\title{
Label-free Imaging of Stem Cell Adhesion and Dynamic Tracking of Boundary Evolution Using Photonic Crystal Enhanced Microscopy (PCEM)
}

\author{
Yue Zhuo ${ }^{1,4}$, Ji Sun Choi ${ }^{2,5}$, Thibault Marin ${ }^{6}$, Hojeong Yu ${ }^{3}$, Brendan A. Harley ${ }^{2,5}$ and Brian T. \\ Cunningham ${ }^{1,3,4}$ \\ ${ }^{1 .}$ Department of Bioengineering, University of Illinois at Urbana-Champaign, Urbana, IL, \\ 2. Department of Chemical and Biomolecular Engineering, University of Illinois at Urbana-Champaign, \\ Urbana, IL \\ ${ }^{3 .}$ Department of Electrical and Computer Engineering, University of Illinois at Urbana-Champaign, \\ Urbana, IL \\ 4. Micro and Nanotechnology Laboratory, University of Illinois at Urbana-Champaign, Urbana, IL, \\ 5. Carl R. Woese Institute for Genomic Biology, University of Illinois at Urbana-Champaign, Urbana, IL, \\ USA \\ 6. Atkins Building, Research Park, Champaign, IL, USA
}

Cell adhesion provides structural support and contributes to functional processes crucial for cellular proliferation and survival. We apply a new form of label-free biosensor imaging called Photonic Crystal Enhanced Microscopy (PCEM) to investigate adhesion/migration of stem cells [1-4]. By applying image processing algorithms to analyze the PCEM images, we offer insight into how the cellular membrane is dynamically organized during cell adhesion.

PCEM utilizes a modified brightfield microscope to measure the spatial distribution of peak resonance wavelength (PWV) across a nanostructured Photonic Crystal (PC) biosensor surface with submicron spatial resolution for label-free imaging (Fig. 1c). PCEM enables quantitative dynamic imaging of the Effective Mass Density (EMD) of membrane-associated cell components that attach to the PC (Fig. 1ab).

For tracking cell boundary evolution, we adapted the Level Set Method (LSM). As shown in Fig. 2a-b, the mHAT cell boundary can be tracked (along local normal direction to the cell boundary) and membrane-associated EMD images can be sampled along the cell boundary spatially and temporally. The resulting 2D spatiotemporal maps represent the attached cellular EMD (Fig. 2c), cell boundary velocity (Fig. 2d), and total displacement (Fig. 2e) at each sampling window along the cell plasma membrane and time frames, respectively. From these spatiotemporal maps, many interesting phenomena can be observed to highlight specific aspects of cell adhesion behavior with quantitative measurements. The three maps in pseudocolor (Fig. 2c-e) indicate clearly that the attached cellular membraneassociated EMD exhibits high amplitude at the two sides (near points A and C) of the cell body $\left(\sigma_{\mathrm{A}}{ }^{*}\right.$ and $\left.\sigma_{\mathrm{C}}{ }^{*}\right)$ at an early adhesion stage (e.g. 40 110 mins), and then slightly decreases. Simultaneously, the cell boundary, especially at the right side of the cell body (near point B), moves towards the right, with the velocity $\left(\mathrm{V}_{\mathrm{B}}\right.$ ) initially high but decreasing with time (Fig. 2d), sharing the same trend (decreasing) as the membrane-associated EMD change $\left(\sigma_{\mathrm{A}}{ }^{*}\right.$ and $\left.\sigma_{\mathrm{C}}{ }^{*}\right)$ (Fig. $2 \mathrm{c}$ ). The accumulated changes in the velocity can also be observed as a delayed increase in the cell boundary moving distance $\left(\mathrm{S}_{\mathrm{B}}\right)$ (Fig. 2e). In addition, statistical analysis can be performed on PCEM data, and one example is shown as the mean and standard deviation estimated through the entire boundary, frame-by-frame, along the temporal dimension (Fig. 3a-c). 
From the above analysis, an early-stage cell adhesion/migration model can be considered (Fig. 3d). When a cell is actively binding/attaching to a substrate surface, it may first initialize some preanchoring sites (e.g. points $\mathrm{A} \& \mathrm{C}$ ) to support the whole cell-body's protrusion in the moving-frontier (point B) in order to prepare for subsequent migration in a specific direction. Therefore, the dynamic label-free images acquired in PCEM can be used to develop a cell attachment model, which may help to further uncover the underlying mechanism of active sensing of the micro cellular environment.

References:

[1] Cunningham, B et al, Sensor Actuat B-Chem 81 (2002), p. 316.

[2] Chen, W. et al, The Analyst 138 (2013), p. 5886.

[3] Zhuo, Y. et al, The Analyst 139 (2014), p. 1007.

[4] Zhuo, Y. \& Cunningham, B. T. Sensors (Basel) 15 (2015), p. 21613.

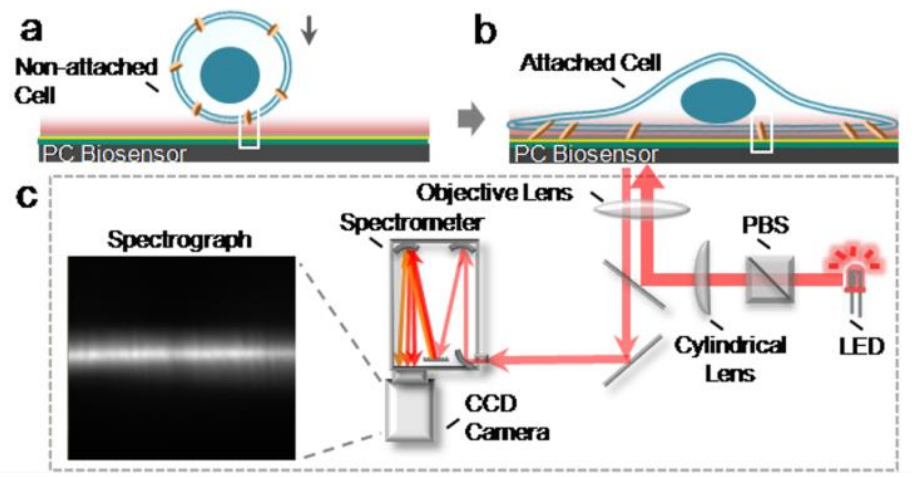

a
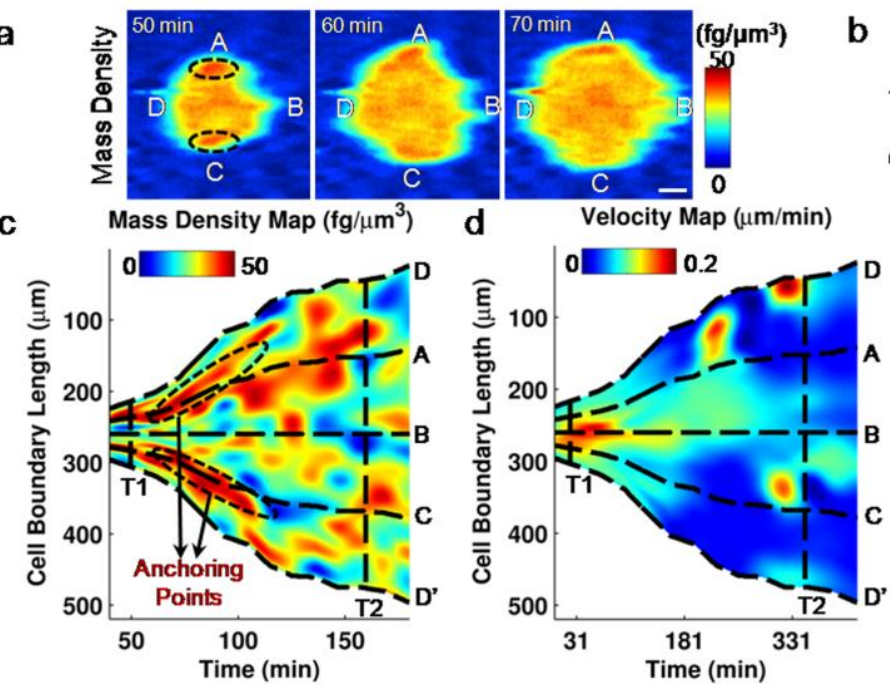

Figure 1. Principle of Cell Adhesion on a PC Slab Biosensor in PCEM. (a) Before cell adhesion: ECM molecules are immobilized on the PC slab biosensor surface. (b) After cell adhesion: Transmembrane mechano-sensitive receptors are activated after adhesion, and focal adhesions are formed on the plasma membrane to form a link between receptors and the cytoskeleton. The effective refractive index is locally increased in each voxel on the PC slab surface. (c) Schematic of the PCEM system.

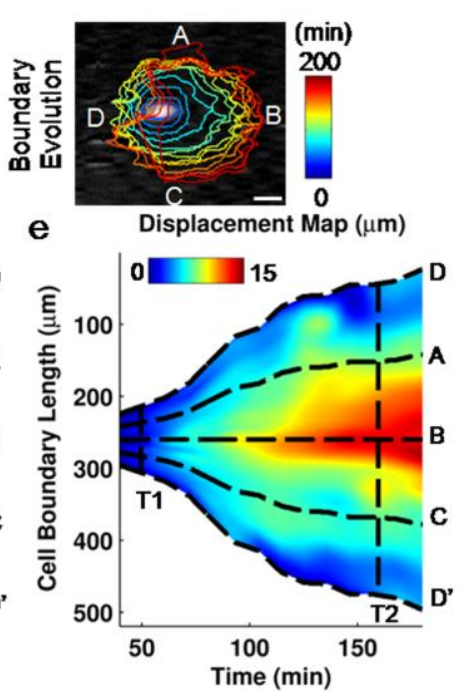

Figure 2. Label-free Imaging with PCEM. (a) EMD images of stem cell (unit: $\mathrm{fg} / \mu \mathrm{m}^{3}$ ). (b) Detected cellular boundaries at different frames (unit: mins). Resampled cellular 2D spatiotemporal maps: (c) EMD map (unit: $\left.\mathrm{fg} / \mu \mathrm{m}^{3}\right), \quad$ Cell boundary (d) velocity map (unit: $\mu \mathrm{m} / \mathrm{min}$ ), and (e) displacement map (unit: $\mu \mathrm{m}$ ). a

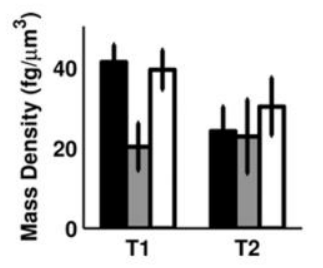

d

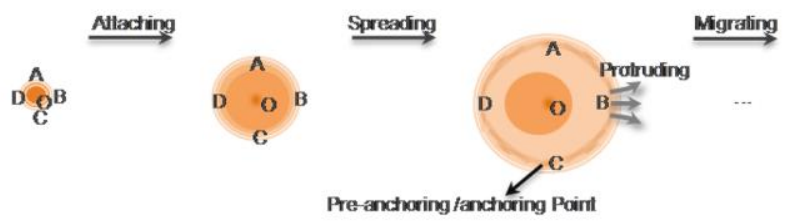

b

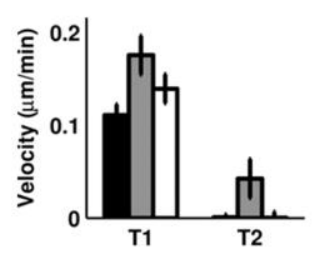

c

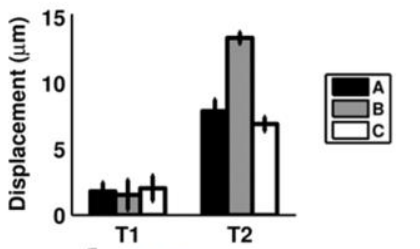

Figure 3. PCEM imaging with statistical analysis (a-c) at different locations $(\mathrm{A}, \mathrm{B}, \mathrm{C})$ along the cell boundary. (d) Hypothesis of earlier status of cell adhesion and migration supported with anchoring points. 